\title{
Arginine deiminase pathway enzymes: evolutionary history in metamonads and other eukaryotes
}

\author{
Lukáš Novák1, Zuzana Zubáčová', Anna Karnkowska ${ }^{1,5}$, Martin Kolisko ${ }^{2,5}$, Miluše Hroudováa ${ }^{3}$ Courtney W. Stairs², \\ Alastair G. B. Simpson ${ }^{4}$, Patrick J. Keeling ${ }^{5}$, Andrew J. Roger ${ }^{2}$, Ivan Čepička ${ }^{6}$ and Vladimír Hampl ${ }^{*}$
}

\begin{abstract}
Background: Multiple prokaryotic lineages use the arginine deiminase (ADI) pathway for anaerobic energy production by arginine degradation. The distribution of this pathway among eukaryotes has been thought to be very limited, with only two specialized groups living in low oxygen environments (Parabasalia and Diplomonadida) known to possess the complete set of all three enzymes. We have performed an extensive survey of available sequence data in order to map the distribution of these enzymes among eukaryotes and to reconstruct their phylogenies.

Results: We have found genes for the complete pathway in almost all examined representatives of Metamonada, the anaerobic protist group that includes parabasalids and diplomonads. Phylogenetic analyses indicate the presence of the complete pathway in the last common ancestor of metamonads and heterologous transformation experiments suggest its cytosolic localization in the metamonad ancestor. Outside Metamonada, the complete pathway occurs rarely, nevertheless, it was found in representatives of most major eukaryotic clades.

Conclusions: Phylogenetic relationships of complete pathways are consistent with the presence of the Archaeaderived ADI pathway in the last common ancestor of all eukaryotes, although other evolutionary scenarios remain possible. The presence of the incomplete set of enzymes is relatively common among eukaryotes and it may be related to the fact that these enzymes are involved in other cellular processes, such as the ornithine-urea cycle. Single protein phylogenies suggest that the evolutionary history of all three enzymes has been shaped by frequent gene losses and horizontal transfers, which may sometimes be connected with their diverse roles in cellular metabolism.
\end{abstract}

Keywords: Arginine deiminase, Ornithine transcarbamylase, Carbamate kinase, Phylogeny, Metamonada, Preaxostyla, Protists

\section{Background}

The arginine deiminase pathway (ADI pathway, syn.: arginine dihydrolase pathway) catalyzes a conversion of arginine to ornithine, ammonium, and carbon dioxide, while generating ATP from ADP and phosphate. The enzymes involved in the three steps of the pathway are arginine deiminase (ADI, EC 3.5.3.6), ornithine transcarbamylase (OTC, EC 2.1.3.3), and carbamate kinase (CK, EC 2.7.2.2). The first reaction, catalyzed by ADI, is the deamination of arginine to yield citrulline and $\mathrm{NH}_{4}^{+}$. OTC then catalyzes

\footnotetext{
* Correspondence: vlada@natur.cuni.cz

'Department of Parasitology, Charles University, Faculty of Science, Prague, Czech Republic

Full list of author information is available at the end of the article
}

the conversion of citrulline and inorganic phosphate into carbamoyl-phosphate and ornithine. Finally, CK catalyzes the hydrolysis of carbamoyl phosphate to form $\mathrm{CO}_{2}$ and $\mathrm{NH}_{4}^{+}$, while the phosphate group is used to regenerate ATP from ADP.

The ADI pathway is widely distributed among bacteria, where it is often a major means of energy production [1]. However, the ammonium produced by this pathway has also been implicated in protecting some bacteria from the harmful effects of acidic environments [2,3]. The pathway has been also described in Archaea [4]. This pathway has only been characterized in a few species of anaerobic eukaryotes namely the parabasalids Trichomonas vaginalis [5] and Tritrichomonas foetus [6], and the diplomonads 
Giardia intestinalis [7], Hexamita inflata [8], and Spironucleus salmonicida [9]. All these species belong to Metamonada (Excavata), a clade of anaerobic protists with substantially modified mitochondria designated as hydrogenosomes or mitosomes. Metamonada consists of three lineages - Fornicata (e.g., Giardia and Spironucleus), Parabasalia (e.g., Trichomonas and Tritrichomonas), and finally Preaxostyla (Trimastix, Paratrimastix [10], and oxymonads) [11]. Currently, there is no information about the ADI pathway in Preaxostyla.

In Trichomonas vaginalis the ADI pathway generates up to $10 \%$ of the energy produced by glucose fermentation [12]. OTC and CK were shown to be cytosolic, while ADI was described as membrane-associated in both Trichomonas vaginalis and Tritrichomonas foetus [6]. The ADI of Trichomonas vaginalis was later shown to be localized in hydrogenosomes and an in situ $\mathrm{pH}$ buffering function has been proposed [13]. The ADI pathway of Giardia intestinalis is completely cytosolic and produces up to 8 times more ATP than sugar metabolism [7]. Besides this energyproducing function, it has been proposed that the enzymes play an important role in the pathogenesis of Giardia intestinalis and Trichomonas vaginalis. The protists secrete ADI and OTC from their cells causing arginine depletion thus reducing the ability of the infected tissue to produce antimicrobial nitric oxide $[14,15]$. Other known effects of these parasite's ADI pathway enzymes include growth arrest of intestinal epithelial cells [16], inhibition of $\mathrm{T}$ cell proliferation [15], and alteration of the phenotype and cytokine production of dendritic cells [17]. Another diplomonad with a characterized ADI pathway, the free-living Hexamita inflata, inhabits environments with varying levels of dissolved oxygen. It has been suggested that the ADI pathway may contribute to the metabolic flexibility of this organism, producing a significant amount of ATP under oxygenlimited conditions, while glycolysis is the main energy source under oxic or microoxic conditions, however the oxygen relationship might be incidental or secondary [8].

Of the three enzymes, only ADI itself is considered to be specific to the ADI pathway. CK has an additional role in purine and nitrogen metabolism and OTC may catalyze synthesis of citrulline as a nitrogen reservoir in plants [18] or be a part of ornithine-urea cycle in animals, diatoms and dinoflagellates $[19,20]$. Therefore, the presence of ADI in organisms where no ADI pathway is known is intriguing and deserves further investigation. For example, within the chlorophytes the ADI gene was found in three species of Chlorella [21, 22] and Chlamydomonas reinhardtii [23] And ADI activity has been reported in multiple species of Chlorodendrophyceae, Trebouxiophyceae, Chlorophyceae, and Ulvophyceae [24], that is, in all classes of the "crown group" of Chlorophyta [25].

The first known sequence of a eukaryotic ADI, from Giardia intestinalis, showed no specific relationship to any bacterial or archaeal clade [26]. Later analyses included sequences from Trichomonas vaginalis, Spironucleus vortens, Sp. barkhanus, and Sp. salmonicida (Metamonada), Euglena gracilis and 'Seculamonas' sp. (Discoba), Chlamydomonas reinhardtii and Chlorella sp. (core Chlorophyta [27]), and Mastigamoeba balamuthi and Dictyostelium discoideum (Amoebozoa). All the eukaryotic sequences formed a wellsupported clade related to Archaea, consistent with a single origin of ADI in the eukaryotic domain $[9,13]$.

Due to its involvement in other pathways, it is not surprising that OTC is more widespread among eukaryotes compared to the other ADI pathway enzymes. The phylogenetic analysis of OTC by Zúñiga et al. [26] recovered two distinct eukaryotic clades branching in different positions among bacteria, one comprising sequences from Embryophyta and other composed of metazoan and fungal sequences. The only eukaryote outside these two clades was Giardia intestinalis, which was also the only one with a characterized ADI pathway. The sequence from Giardia intestinalis branched among bacterial sequences without close relationship to any other eukaryotic clade. Later analyses demonstrated that Spironucleus salmonicida and Trichomonas vaginalis OTC sequences formed a wellsupported clade with Giardia intestinalis [9], suggesting the existence of a third independent group of eukaryotic OTCs present in Metamonada and potentially involved in the ADI pathway. The same analysis also showed two stramenopile sequences branching clearly inside the Metazoa-Fungi group.

Sequences of CK from Giardia intestinalis, Hexamita sp., and Trichomonas vaginalis formed a relatively wellsupported clade not closely related to any bacterial or archaeal sequences [26]. The monophyly of eukaryotic CKs was later questioned after adding sequences from Spironucleus salmonicida and Carpediemonas membranifera, with the Trichomonas vaginalis sequence branching separately from other eukaryotes, although statistical support for this topology was very low [9].

In summary, the complete set of ADI pathway enzymes has been found in representatives of two out of three major lineages of Metamonada: Parabasalia and Fornicata. All the metamonad enzymes appear to be closely related to each other. This raises several questions about the evolutionary history of the pathway among eukaryotes. Is it present also in the third and least investigated lineage of metamonads, Preaxostyla? Was it present in the common ancestor of the Metamonada? Do representatives of other eukaryotic lineages possess the ADI pathway as well? If so, do all the eukaryotic enzymes involved in the ADI pathway originate from the same source or do they represent independent acquisitions?

Here, we take advantage of the recent progress in genome and transcriptome sequencing of less studied protists to perform an up-to-date survey and 
phylogenetic analysis of ADIs, OTCs, and CKs. This survey focuses on elucidating the evolutionary history of the arginine deiminase pathway in eukaryotes, with special emphasis on Metamonada. In addition to phylogenetic studies, we determine the subcellular localization of these enzymes in two members of Preaxostyla, Paratrimastix pyriformis and oxymonad Monocercomonoides sp. PA203.

\section{Results}

Distribution of ADI, OTC, and CK across eukaryotes

Our survey revealed the presence of ADI, OTC, and CK in the three main eukaryotic clades defined by Adl et al., 2012 [28] (Fig. 1). The first and presumably the most specific enzyme of the pathway, i.e. without any role outside the ADI pathway reported so far, is ADI itself. This was found in 40 taxa, as shown on the schematic tree in

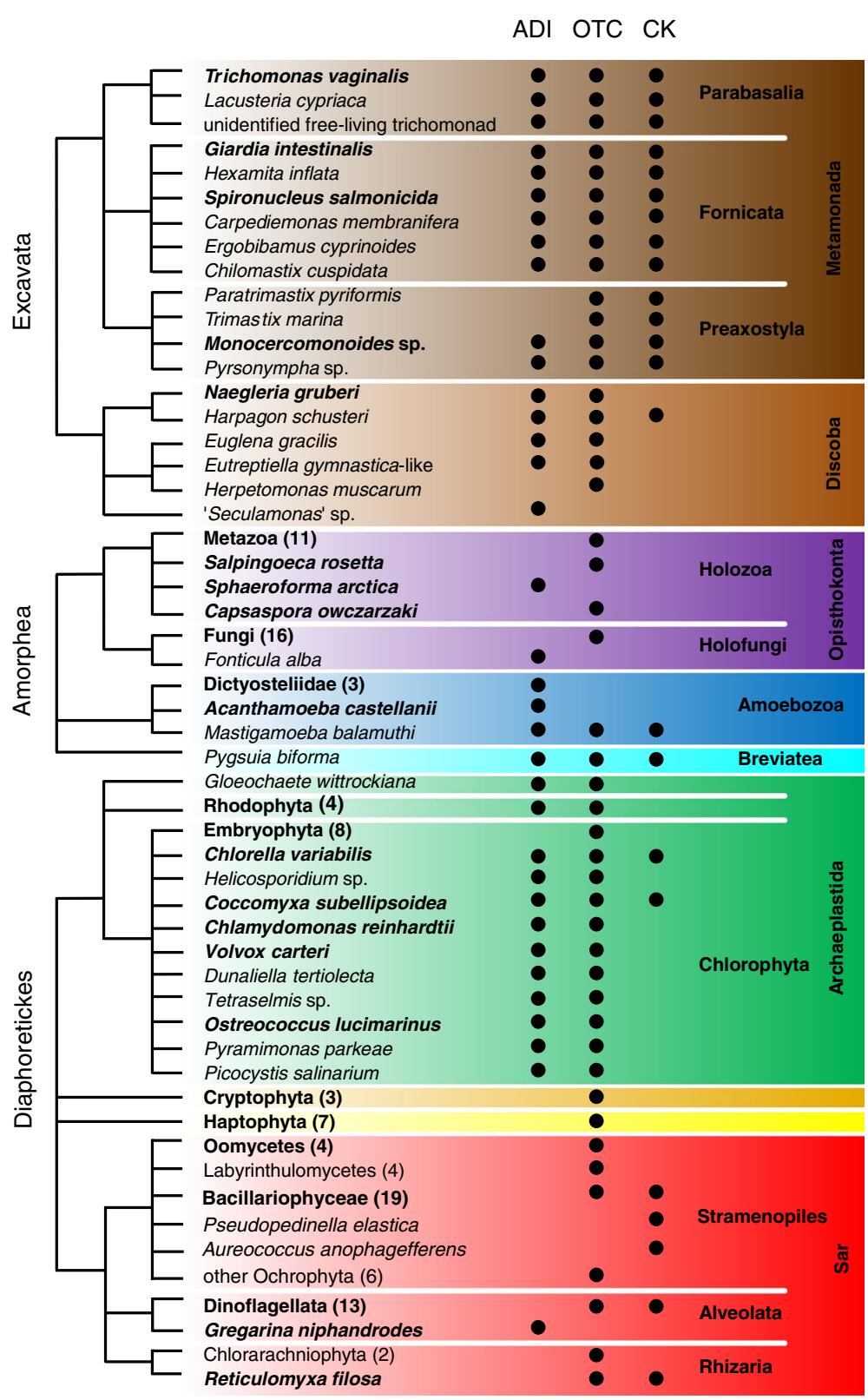

Fig. 1 Distribution of enzymes of the arginine deiminase pathway across eukaryotic diversity. Arginine deiminase (ADI), ornithine transcarbamylase (OTC), carbamate kinase (CK). Taxon names in boldface indicate lineages containing at least one representative with a sequenced nuclear genome. Numbers in brackets indicate number of sequences from the given taxon included in our analyses. Colored rectangles indicate major eukaryotic groups as follows: dark brown - Metamonada; light brown - Discoba; violet - Opisthokonta; blue - Amoebozoa; cyan - Breviatea, green - Archaeplastida; orange - Cryptophyta; yellow - Haptophyta; red - SAR. Excavata, Amorphea, and Diaphoretickes are names of the 3 putative largest clades of eukaryotes as proposed in Adl et al., 2012; relationships between them are not resolved 
Fig. 1, and these taxa represent most eukaryotic supergroups (highlighted by colored backgrounds). Of these, 16 species (most metamonads, Harpagon, Mastigamoeba, Pygsuia, Chlorella, and Coccomyxa) encoded all three enzymes, while the other species encoded only one or two enzymes. ADI was not detected in any representative of the clades Metazoa, Fungi, Embryophyta, Cryptophyta, and Haptophyta, nor in Sar [28], with the single questionable exception of Gregarina niphandrodes (see below). OTC was the most widespread enzyme, being found in 131 taxa including the major multicellular groups of Metazoa, Fungi, and Embryophyta. CK was detected in all the investigated metamonads, multiple Bacillariophyceae, Dinoflagellata and 8 other species. Please note that the given numbers do not represent the actual quantity of eukaryotic species with the particular gene since several groups, e.g. Metazoa, Bacillariophyceae, are represented by only a limited number of randomly selected sequences.

\section{Phylogenetic analyses \\ Arginine deiminase}

Compared to the previous analyses we present a more robust analysis including 40 eukaryotic species (Fig. 1). The phylogenetic tree (Fig. 2) shows two clearly separated (RAxML bootstrap support/IQ-TREE bootstrap support: $100 \% / 100 \%)$ clans of ADIs, one comprising all bacteria and one isolated eukaryote, Gregarina niphandrodes, in a highly nested but poorly resolved position, and the second composed of clans of Archaea plus a few Bacteria (64 \%/ $97 \%)$ and Eukaryota (51 \%/81 \%). The topology within the eukaryotic branch is poorly resolved overall, however, a few clades of lower-than-supergroup rank were recovered with strong support (i.e. bootstrap support $>80 \%$ ). These are Parabasalia, Diplomonadida, Oxymonadida, Chlorophyta, and Dictyosteliida.

\section{Ornithine transcarbamylase}

Our analysis included sequences from selected representatives of Metazoa, Fungi, and Embryophyta and 110 sequences from 103 other eukaryotes (Fig. 1). Several bacterial sequences of aspartate transcarbamylase, a protein closely related to OTC, were included to provide an outgroup for rooting the tree. Our analysis of OTC phylogeny (Additional file 1) supports the existence of three large groups and two separately-branching eukaryotic OTCs.

The first large clade is strongly supported (100\%/100\%) and contains Metazoa, Fungi, Oomycota, Bacillariophyceae (i.e. diatoms), a few lineages of other Stramenopiles (Nannochloropsis, Vaucheria, Ectocarpus, Heterosigma and Ochromonas), the holozoan Capsaspora, and one single excavate, the trypanosomatid Herpetomonas muscarum, which branches among Fungi.
The second eukaryotic group, already indicated in the analysis by Andersson et al. [9], is well supported (83 \%/ $100 \%)$ and includes all Metamonada, Harpagon shusteri, Naegleria gruberi, Reticulomyxa filosa, Pygsuia biforma, Mastigamoeba balamuthi, and many representatives of autotrophic groups, namely dinoflagellates, cryptophytes, euglenophytes, chlorarachniophytes, and stramenopiles like Aureococcus, Aureoumbra, Pelagococcus, Pelagomonas, and Pseudopedinella. The monophyly of Parabasalia is well supported. A sequence from a recently described archaeon Lokiarchaeum sp. is also included in this group, however at an unsupported position.

The third group is composed of euglenophytes, green algae with green plants, red algae, and haptophytes, with haptophytes branching inside red algae. A clade of mostly Desulfobacteraceae bacterial sequences branches inside this group of eukaryotic sequences.

The only two eukaryotes outside these three large clades are the choanoflagellate Salpingoeca rosetta (sequence obtained from the genome), which branches as sister to Microgenomates bacterium (78 \%/100 \%), and the rhizarian Paulinella chromatophora (red star in Additional file 1) inside Cyanobacteria with good statistical support (100 \%/ $100 \%)$. Since the Paulinella sequence originates from the genome of the chromatophore, not the Paulinella nucleus, it actually represents a cyanobacterial OTC.

\section{Carbamate kinase}

We have included 47 sequences from 44 eukaryotic species in our analysis (Fig. 1). Our tree (Additional file 2) shows eukaryotes falling into several separate clusters. One of two substantial groups is an unsupported clan of Fornicata, Parabasalia, Harpagon schusteri, Pygsuia biforma, and Mastigamoeba balamuthi. A well-supported Preaxostyla clade (96\%/100 \%) branches at a different place among bacteria, as a sister group to Hadesarchaea archaeon and Anaerolineae bacterium (96\%/100 \%). The second large eukaryotic clan $(100 \% / 100 \%)$ is composed of all the dinoflagellate sequences, as well as sequences from diatoms, Pedinella and Aureococcus. Dinoflagellata form a well-supported group within this clan. Three sequences from diatoms do not branch together with other ochrophytes (the photosynthetic Stramenopiles), and instead form a separate well-supported clan $(100 \% / 100 \%)$ among bacteria. This may represent a second form of the enzyme, since Thalassiosira pseudonana appears in both diatom groups. The only two known CKs from green plants (Chlorella and Coccomyxa) branch together (97\%/99 \%) but separated from other eukaryotes. The Reticulomyxa CK sequence is also isolated from the rest of eukaryotes.

\section{Concatenation}

We also performed a phylogenetic analysis of a concatenation of all three enzymes. In the first step, we have 


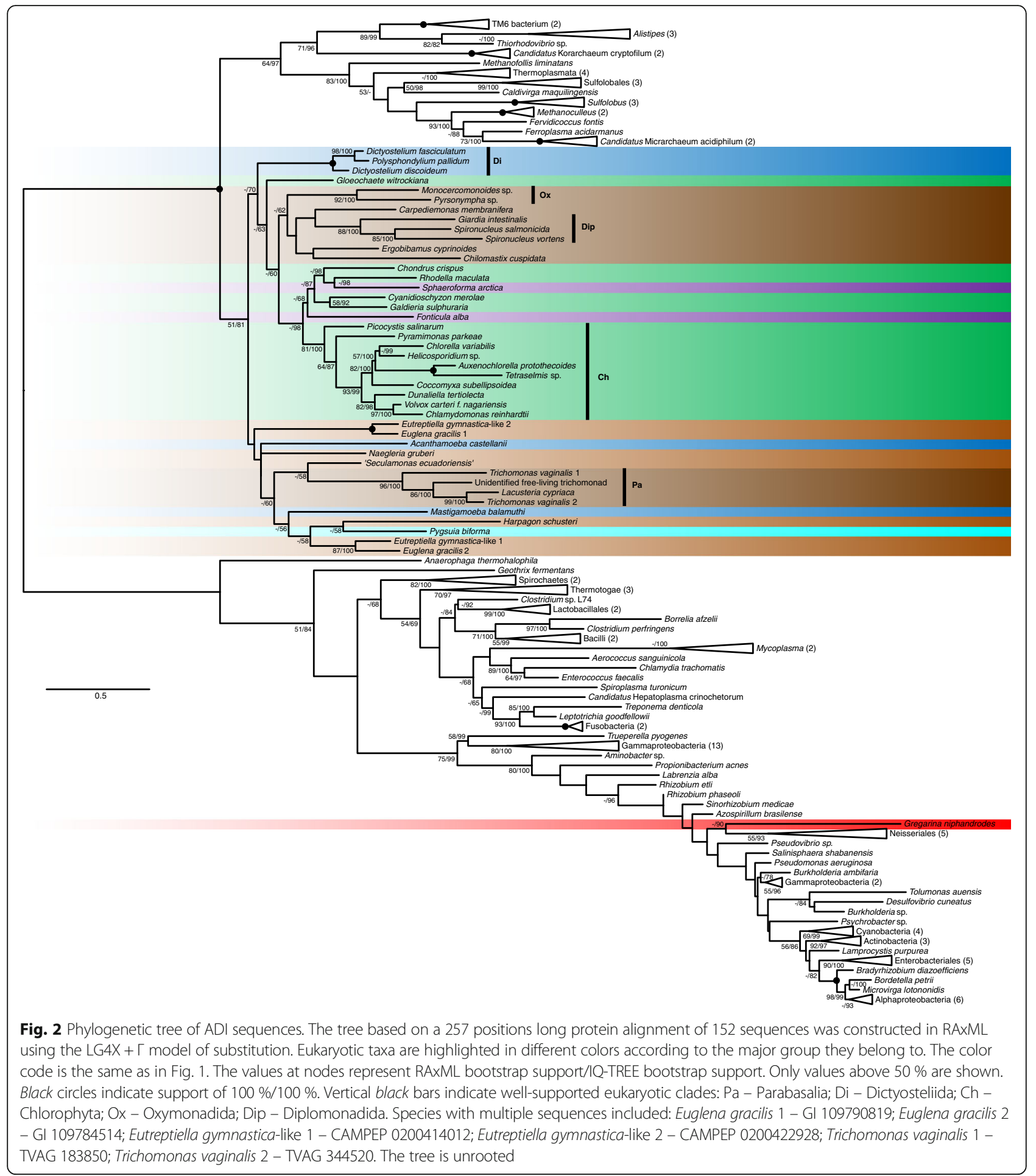

prepared an alignment supermatrix in which we have included all eukaryotes and representatives of prokaryotes that contain a complete set of the three enzymes, and may use the ADI pathway. In order to detect potential incongruities between gene partitions caused by lateral gene transfer we have performed a phylogenetic analyses of the individual gene partitions from this supermatrix. Based on these gene trees (Additional file 3) we removed taxongene sequences that branched with bootstrap support higher than $50 \%$ within a clan of sequences outside its own domain (e. g. eukaryotic sequence outside Eukaryota) from the concatenated alignment - namely CKs from 
Monocercomonoides sp., Pyrsonympha sp., Chlorella variabilis, and Coccomyxa subelipsoidea. We also removed OTCs from Chlorella variabilis, and Coccomyxa subelipsoidea because in the large single gene tree (Additional file 1) they branch within a clade which is sister to Chlorobi Bacteria with $100 \%$ IQ-TREE bootstrap support.

The analysis performed on the alignment after removal of these sequences (Additional file 4) revealed a strong bipartition (100 \%/100 \%) grouping Eukaryota and Archaea to the exclusion of Bacteria and within this part of the tree the eukaryotes formed a well-supported (100\%/ $100 \%$ ) clan sister to the archaeon Candidatus Korarchaeum cryptofilum. In order to recover the relationships within the Eukaryota-Archaea group without the disturbing long branch of Bacteria we repeated the analysis without the bacterial sequences (Fig. 3). In this unrooted tree Eukaryota are grouped with Candidatus Korarchaeum cryptofilum to the exclusion of the rest of Archaea with high support (97\%/98 \%). We also performed a Eukaryota-only analysis of the concatenated dataset for the purpose of hypotheses testing (Additional file 5).

\section{Hypotheses testing}

We used approximately unbiased (AU) and expected likelihood weight (ELW) tests to assess whether the inferred phylogenies are in a significant conflict with the monophyly of eukaryotes, metamonads and with the expected eukaryotic species tree. The results are summarized in Table 1 . The AU tests rejected monophyly of metamonads in the OTC and CK trees and monophyly of expected eukaryotic phylogeny in concatenation. The ELW tests rejected the monophyly of metamonads in the OTC tree, the monophyly of both clades in the CK and monophyly of metamonads and the expected eukaryotic phylogeny in the concatenation tree.

\section{Localization of ADI pathway enzymes in Preaxostyla}

Another aim of this work was to infer the subcellular localization of ADI pathway enzymes in members of the poorly studied Preaxostyla clade. Genomic and transcriptomic projects have revealed the presence of all three enzymes in Monocercomonoides sp. and Pyrsonympha sp., while only OTC and CK enzymes were detected in Trimastix marina and Paratrimastix pyriformis. We have chosen Monocercomonoides sp. PA203 and Paratrimastix pyriformis for further study.

We investigated the presence of mitochondrion-targeting signals in the enzymes of interest (Additional file 6) using the signal prediction software TargetP 1.1 [29] and Mitoprot II v1.101 [30]. TargetP did not predict any targeting signals. Mitoprot II predicted a single mitochondrion-targeting signal, for the OTC sequence of Paratrimastix pyriformis.

To validate the results of mitochondrion-targeting signal prediction we used the Trichomonas vaginalis $\mathrm{T} 1$

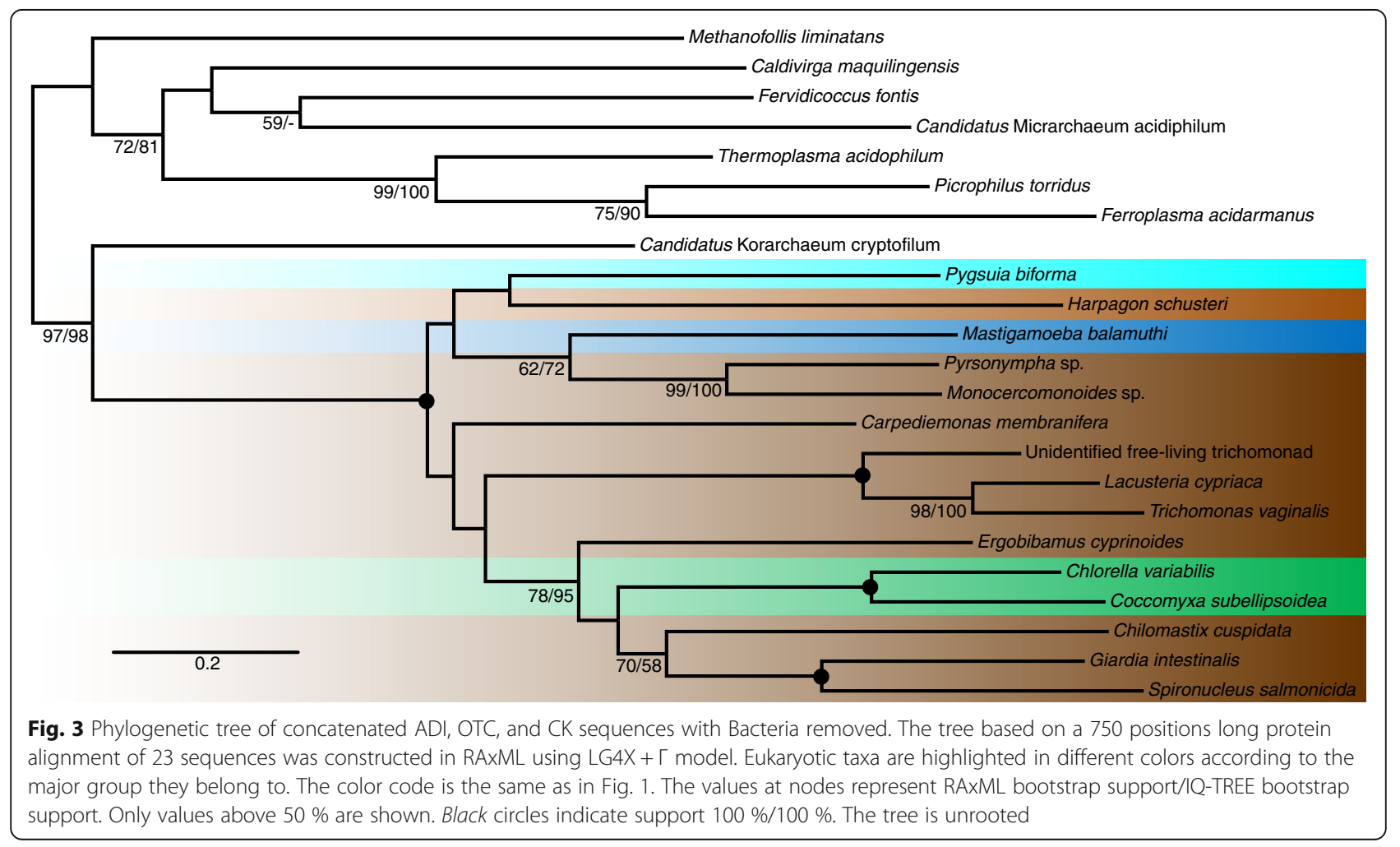


Table 1 Results of approximately unbiased (AU) and expected likelihood weights (ELW) tests

\begin{tabular}{lll}
\hline Data set - hypothesis & AU test & ELW test \\
\hline ADI - Metamonada monophyly. & 0.64 & 0.10 \\
OTC - Eukaryota monophyly & 0.64 & 0.5 \\
OTC - Metamonada monophyly & 0 & 0 \\
CK - Eukaryota monophyly & 0.12 & 0 \\
CK - Metamonada monophyly & 0 & 0 \\
Euk. Conc. - Metamonada monophyly & 0.17 & 0 \\
Euk. Conc. - expected euk. phylogeny & 0 & 0
\end{tabular}

The tests were performed for 4 sets of taxa - ADI: ADI dataset (as in Fig. 2), OTC: OTC dataset (as in Additional file 1), CK: CK dataset (as in Additional file 2), Euk. Conc.: concatenation dataset without prokaryotic sequences (as in Additional file 5)

heterologous expression system, with the assumption that an undetected mitochondrion-targeting signal may nonetheless be recognized by the Trichomonas hydrogenosomal import machinery. We transfected Trichomonas vaginalis cells with plasmids containing HA-tagged OTC and CK from Paratrimastix pyriformis and ADI, OTC, and CK from Monocercomonoides sp. In all cases fluorescence microscopy showed that the heterologously expressed proteins do not co-localize with the signal from the hydrogenosomal marker protein (malic enzyme), but instead formed a diffuse pattern all over the cell (Fig. 4). This demonstrates that the inserted proteins are not recognized as hydrogenosomal-import targets in Trichomonas vaginalis. The results of these experiments are consistent with the fact that most ADI pathway enzymes in eukaryotes are localized in the cytosol.

\section{Discussion}

\section{ADI pathway enzymes are widespread in eukaryotes}

Before this study, only two closely related lineages of eukaryotes had been conclusively shown to possess a complete ADI pathway. These were Parabasalia and Diplomonadida, both being members of Metamonada, a subgroup of Excavata. Our survey has shown the presence of all three enzymes in ten other eukaryotic species. Among these are other members of Metamonada - including free-living members of Fornicata related to the predominantly parasitic diplomonads (Ergobibamus cyprinoides, Chilomastix cuspidata, Carpediemonas membranifera), and members of the third metamonad lineage, Preaxostyla (Monocercomonoides sp. and Pyrsonympha sp.). The ADI pathway was also identified in nonmetamonads including the heterolobosean Harpagon schusteri, the amoebozoan Mastigamoeba balamuthi, the breviate Pygsuia biforma, and the green algae Chlorella variabilis and Coccomyxa subelipsoidea. Further functional studies are needed to determine whether these enzymes function within an ADI pathway in these species. It is possible that the possession of the complete pathway is connected with their anaerobic lifestyle since most of these organisms are anaerobes, microaerophiles or aerobes able to live for long periods under anaerobic conditions [31-37].

Many investigated eukaryotes possessed incomplete sets of ADI pathway enzymes. The presence of OTC or CK on their own is not surprising, as they are known to be involved in other biochemical processes including the ornithine-urea cycle or purine biosynthesis. The presence of ADI on its own was unexpected, yet we identified ADI in a broad spectrum of eukaryotic lineages without complete pathway. The apparent absence of OTC or CK may be due to the incompleteness of transcriptome or genome data, nevertheless, our observation suggests that ADI may also function outside the context of the ADI pathway in some eukaryotes.

\section{Phylogenetic histories of enzymes}

None of the enzyme phylogenies is completely consistent with the expected species relationships. In single gene trees, eukaryotes are always dispersed in multiple clades, suggesting complicated evolutionary histories. The backbone topologies were generally weakly supported, and many of these incongruences are probably the result of low phylogenetic signal. Nevertheless, some conflicts with species phylogeny are better supported and some were confirmed by phylogenetic tests. These can potentially be attributed to lateral gene transfers (LGTs, also known as horizontal gene transfers - HGTs) or endosymbiotic gene transfers (EGTs). The sister relationship of Preaxostyla and Spirochaeta in the CK tree, and the position of Gregarina within Bacteria in the ADI tree are two such examples of potential LGT, albeit the latter may also represent a contamination. Since haptophytes are known to harbor secondary plastids of potentially red algal origin [38], the position of haptophytes within a red algal clade in the OTC analysis, might represent a potential EGT. Conversely, many moderately and robustly supported eukaryotic clades are taxonomically reasonable, indicating the important role of vertical inheritance.

The taxon sampling in the concatenation analyses was lower, because the analyses included only those taxa that may utilize the arginine deiminase pathway and not those that use individual enzymes for other purposes. The resolution of the concatenation tree was higher than the individual gene trees and strongly supported the monophyly of eukaryotes $(99 \% / 100 \%)$ and their close relationship to Archaea. The clade of eukaryotes branched with Archaea (100 \%/100 \%) as a sister to Candidatus Korarcheum cryptophylum. Increased support of these nodes should partly be ascribed to the lower number of taxa but it also suggests that the phylogenetic signal regarding these deep nodes for this narrow set of taxa and after exclusion of obvious cases of LGT is largely congruent. 

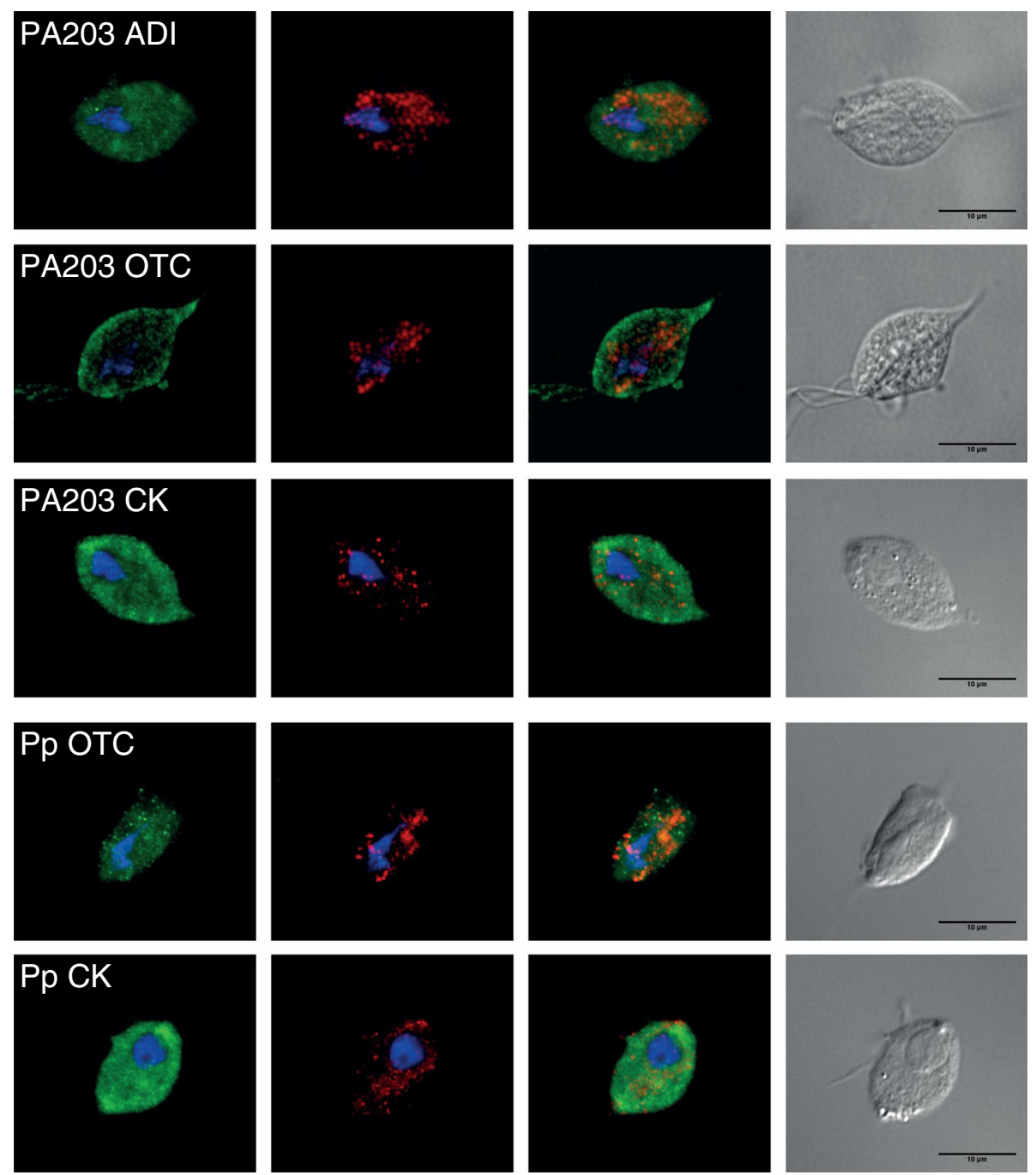

Fig. 4 Localisation of Monocercomonoides and Paratrimastix enzymes in Trichomonas vaginalis cells. Immunofluorescence micrographs of Trichomonas vaginalis, in which the HA-tagged versions of enzymes were expressed. Green signal from anti-HA antibody does not localize to hydrogenosomes of Trichomonas vaginalis, which are marked by red anti-malic enzyme antibody. Blue signal indicates DAPI-stained nuclei. Abbreviations: PA203 - Monocercomonoides sp. PA203; Pp - Paratrimastix pyriformis; ADI - arginine deiminase; OTC - ornithine transcarbamylase; CK carbamate kinase

\section{ADI pathway is ancestral in Metamonada}

The presence of the complete ADI pathway is widespread in metamonads, protists that specifically inhabit low-oxygen environments. In most of the phylogenies, metamonad taxa branch close to each other, but they never form an exclusive clade. In CK trees there is a wellsupported relationship between Preaxostyla (Paratrimastix, Monocercomonoides, and Pyrsonympha) sequences and a prokaryotic clan of Hadesarchaea archaeon and Anaerolineae bacterium. Metamonada do not appear monophyletic even in the concatenation trees and their monophyly was rejected by ELW test. Taking together all this information we propose that the complete arginine deiminase pathway was present already in the common ancestor of Metamonada and was vertically inherited by the extant metamonad lineages, with a few exceptions. The exceptions are the putative losses of ADI in Trimastix marina and Paratrimastix pyriformis and the putative replacement by a bacterial CK in the Preaxostyla. It is also possible that some enzymes or the whole pathway were laterally transferred from metamonads to other eukaryotes, which would disrupt the monophyly of Metamonada on trees.

Our localization experiments suggest that all enzymes in both Paratrimastix pyriformis and Monocercomonoides sp. are localized in the cytosol, like the enzymes in Giardia intestinalis but unlike the ADI in Trichomonas vaginalis. We therefore conclude that cytosolic localization of the pathway is an ancestral trait of all metamonads. It should be noted, however, that the cytosolic localization of ADI in 
Monocercomonoides sp. may not be informative, since this protist does not contain mitochondrion [39]. Paratrimastix pyriformis harbors an organelle similar to the hydrogenosome, but no ADI-coding gene has been found in its transcriptome to test its localization.

\section{ADI pathways in other eukaryotes}

Besides Metamonada, five other species contain a complete ADI pathway and all branch within the eukaryotic clade on the concatenation tree (Fig. 3). These represent four different supergroups of eukaryotes; only Harpagon is from the same supergroup as Metamonada (supergroup Excavata), and even then it belongs to a different sub-branch (Discoba). Moreover, Excavata are likely not monophyletic at all [40] and the root of eukaryotes may be situated inside the group. Harpagon, Mastigamoeba, and Pygsuia ADI pathway enzymes branch close to Metamonada in all three gene trees, and so it is very probable that the pathway in these three species was derived from the same source as the pathway in Metamonada.

The situation in Chlorella variabilis and Coccomyxa subelipsoidea is less clear. These organisms are the only green algae known to contain all three ADI pathway enzymes. Their ADIs and OTCs branch together with other green algae and plants in the individual gene trees (while no other Plantae have CK), supporting the presence of the complete set in the last common ancestor of Chlorophyta. However, Chlorophyta did not branch in a common eukaryotic clade with Metamonada, Harpagon, Mastigamoeba and Pygsuia in OTC and CK phylogenies, suggesting that these two enzymes in Chlorophyta may have independent origins. In the concatenation tree, Chlorella variabilis and Coccomyxa subelipsoidea represented only by ADI sequences branch together with other eukaryotes. Presence of the ADI pathway in the last common ancestor of Chlorophyta would be consistent with the ADI enzymatic activity previously reported from members of Chlorodendrophyceae, Trebouxiophyceae, Chlorophyceae, and Ulvophyceae [24]. However, the function of the other enzymes in a typical ADI pathway is questionable, since the OTCs from Chlorella autotrophica, Chlorella saccharophila (Trebouxiophyceae), and Dunaliella tertiolecta (Chlorophyceae) were found to have no measurable activity in the direction of the ADI pathway, i.e. conversion of citrulline to ornithine [24]. It is therefore possible that the two Chlorophyta species with all three enzymes nonetheless do not use the ADI pathway.

\section{Origin of the eukaryotic ADI pathway}

The simplest explanation of the fact that the complete sets of ADI pathway enzymes from several eukaryotic lineages are related is that they are inherited from their common ancestors. The taxonomic composition of the eukaryotic clade in the concatenation tree is so broad that their common ancestor must have been either the last eukaryotic common ancestor (LECA) or its close descendant. This assumption is reasonable even if we would not consider Chlorella and Coccomyxa. An alternative explanation for the close relationships of ADI pathways would be that the genes were acquired more recently by one eukaryotic lineage (perhaps Metamonada, where it is most common), and then spread from this lineage into others via eukaryote-to-eukaryote lateral gene transfers.

Based on our data we are unable to decide which alternative is more likely. Vertical inheritance of the ADI pathway from LECA would be consistent with the sisterhood of the eukaryotic clade and the archaeon Candidatus Korarchaeum cryptofilum in the concatenation tree (Fig. 3, Additional file 4), since recent studies indicate Korarchaeota are indeed closely related to the eukaryotes [41]. Moreover, OTC sequences from Lokiarchaeum sp., which is the closest known relative to eukaryotes [42], are related to the Metamonada-containing eukaryotic clade. CK sequence of this archaeon branches outside eukaryotes sister to a proteobacterium Desulphobacula toluolica (97 \%/ $100 \%$ ), but nodes separating these two from Metamonada did not receive strong support. ADI sequence from Lokiarcheum is not available, and so this organism was not included in the concatenation analysis. We must also take into account that the position of the root of the eukaryotic tree is still an unresolved question [40, 43, 44]. If the root falls outside Amorphea + Discoba (position of Metamonada relative to the root is not known), then our results do not necessarily uncover the condition of LECA.

The later acquisition of the pathway by Metamonada and its spread to unrelated eukaryotes by eukaryote-toeukaryote transfer is supported by the fact that the concatenation trees are incongruent with the expected relationships of taxa.

\section{Conclusions}

Our broad survey of the arginine deiminase pathway enzymes has shown that they are present in representatives of all major lineages of eukaryotes. Sixteen protists (most metamonads, Harpagon, Mastigamoeba, Pygsuia, Chlorella, and Coccomyxa) contain the complete set of the three enzymes, while other organisms contain incomplete sets. The enzyme ADI is present in several species without the complete arginine deiminase pathway, suggesting its involvement in other cellular processes. The topology of individual gene trees is generally not very well supported and particularly in OTC and CK trees the eukaryotic enzymes form multiple clearly unrelated clades consisting of mixtures of eukaryotic supergroups. This indicates that multiple prokaryote-to-eukaryote and eukaryote-to-eukaryote LGT events took place in the history of these enzymes. It is possible that in some groups the enzyme acquisition was 
connected with its involvement in novel biochemical processes like the ornithine-urea cycle.

Based on the presence of the complete pathway in most metamonads and based on the phylogenetic affinity of metamonad enzymes, we conclude that the ancestor of metamonads already possessed this pathway. The concatenation analyses suggest that eukaryotes with the complete ADI pathway, including Metamonads, Harpagon, Mastigamoeba and Pygsuia (and possibly Chlorella and Coccomyxa), may have acquired the genes from a single, archaeon-related source. One intriguing possibility is that the acquisition of the pathway may date back as deep as to LECA, but other scenarios involving LGT events are also plausible. To resolve the last issue, it will be necessary to obtain data from a more diverse set of prokaryotes and eukaryotes, especially those branching close to the root of the eukaryotic tree and close to the root of major eukaryotic lineages.

\section{Methods}

\section{Obtaining sequences}

The majority of eukaryotic sequences included in the survey were obtained from the NCBI database (Release 68), JGI database [45], or Marine Microbial Eukaryote Transcriptome Sequencing Project [46]. Initial searches were performed using BLASTp and tBLASTn algorithms [47] with Giardia intestinalis, Trichomonas vaginalis, and several bacterial sequences as queries. The searches of public databases were then repeated several times while restricted to a particular major eukaryotic lineage (e.g. Cryptophyta, Alveolata) and with a phylogenetically closest available sequence as a query. All the eukaryotic sequences with E-value lower than $10^{-3}$ were downloaded and used in subsequent analyses.

The prokaryotic sequences included in the survey were retrieved from the NCBI database using the same query as in the search for eukaryotic sequences. The search was also repeated several times with varying taxonomic restrictions to ensure that all the bacterial and archaeal phyla containing the particular enzyme are represented in the analysis. We used all archaeal sequences with Evalue lower than $10^{-3}$ and a limited number of bacterial sequences with E-value lower than $10^{-3}$ and annotated as the protein of interest. It is important to note that the set of bacterial sequences used in our analyses is not exhaustive and therefore we do not infer any evolutionary hypotheses about Bacteria in this study.

In order to mitigate the risk of missing prokaryotic data influencing the relationships between eukaryotic groups we enriched our datasets with the closest prokaryotic homologs to each of the eukaryotic sequences by searching the NCBI database using BLASTp with each eukaryotic sequence as a query and downloaded the prokaryotic sequence with the lowest e-value from each search.
We investigated whether those eukaryotic sequences which were not branching within eukaryotic clades represent bona fide eukaryotic sequences or contamination of the data sets. Nucleotide sequences obtained from transcriptomic data were checked for similarity with sequences deposited in NCBI and those that were identical or very similar to bacterial genomes (bit score higher than 200 and similarity along the entire length of the sequence) were excluded. In the case of sequences obtained from genomes, the entire gene content of the contiguous sequence scaffold was used as a query for BLAST search of the NCBI database in order to identify any known sequences with high sequence similarity along the entire length of the sequence, indicating possible contamination. Furthermore, candidate sequences and neighboring genes were investigated for the presence of introns and the origin and annotation of surrounding genes. These steps should identify some sequences originating from contamination, however, others could still remain due to the lack of data from the source of the contamination or incorrect assembly of genomic data resulting in chimerical sequences.

The sequences downloaded from public databases were combined with sequences extracted from genomic and transcriptomic projects performed in the laboratories of co-authors. Brief information on the generation of these data sets is given below. Details of the Monocercomonoides sp. PA203 genome and transcriptome project are given in Karnkowska et al. [39]. Details of the Paratrimastix pyriformis transcriptome project are given in Zubáčová et al. [48]. Partial cDNA sequences corresponding to Paratrimastix pyriformis OTC and CK obtained in the transcriptome project were completed at their $5^{`}$ ends by RACE using FirstChoice RLM-RACE kit (Life Technologies, AM1700). Amplifications by PCR were carried out using Takara Hot-Start ExTaq DNA Polymerase (Takara, RR006A) in $50 \mu \mathrm{l}$ reactions. Outer $5{ }^{\prime}$ RLM-RACE PCR was done using the $5{ }^{\prime}$ RACE outer primer supplied in the kit and the following 5 RACE gene-specific outer primers: TpOTCout: CCAGCAGGAAGAGAAGGAGG and TpCKout: GCTTGCCGTAGTTGATGATG. Inner 5 RLM-RACE PCR was done using the 5` RACE inner primer supplied in the kit and the following 5 ' RACE genespecific inner primers: TpOTCinn: AAGAGCTCGTGATCTGGAAG and TpCKinn: GCCAGAGGCGATGACAATGA (here and elsewhere, all primers reported in the 5 'to 3' direction). The following touchdown program was used for each of the two PCRs: $95^{\circ} \mathrm{C}(5 \mathrm{~min}), 15$ cycles of $95{ }^{\circ} \mathrm{C}(1 \mathrm{~min}), 60{ }^{\circ} \mathrm{C}$ to $45{ }^{\circ} \mathrm{C}\left(35 \mathrm{~s}\right.$.) and $72{ }^{\circ} \mathrm{C}(2 \mathrm{~min})$, 20 cycles of $95^{\circ} \mathrm{C}(1 \mathrm{~min}), 45^{\circ} \mathrm{C}(35 \mathrm{~s}$.$) and 72{ }^{\circ} \mathrm{C}(2 \mathrm{~min})$, then a final polymerization step at $72{ }^{\circ} \mathrm{C}$ for $6 \mathrm{~min}$. PCR products were cloned into pGEM-T Easy plasmid vector (Promega, A1360) and sequenced.

Sequences from Lacusteria cypriaca (strain LAI), an unidentified free-living trichomonad (strain LAGOS2D) and 
Pyrsonympha sp. were mined from RNA-seq data sets generated using the llumina MiSeq sequencing platform. Sequences from Trimastix marina, Carpediemonas membranifera, Chilomastix cuspidata, Mastigamoeba balamuthi, and Pygsuia biforma were mined from RNA-seq data sets generated using the Illumina HiSeq sequencing platform. Sequences from Harpagon schusteri were mined from RNA-seq data obtained using the 454 sequencing platform and sequences of Ergobibamus cyprinoides were mined from data sets generated by combination of Sanger and 454 sequencing platforms. The assembled sequences were submitted to GenBank under accession numbers KT883858-KT883885.

\section{Phylogenetic analyses}

Inferred amino acid sequences were aligned using MAFFT version 7 [49] and the resulting alignments were manually trimmed. Highly variable misaligned sections of several eukaryotic sequences, possibly results of sequencing errors, were removed from the alignment manually. The concatenated alignment was constructed from the single gene alignments using SequenceMatrix [50]. The final alignments can be downloaded from our web page: http:// protistologie.cz/hampllab/NovakData.zip [51]. Phylogenetic inference was performed using substitution models suggested by ProtTest 2.4 server [52] - LG4X $+\Gamma$ model. Maximum Likelihood trees were inferred using RAxMLHPC2 version 8 available on CIPRES [53], with 10 starting trees and also using IQ-TREE v1.4.2 [54] under the LG + $\mathrm{C} 20+\mathrm{F}+\mathrm{G} 4$ model for the single gene trees and LG + $\mathrm{C} 40+\mathrm{F}+\mathrm{G} 4$ model for concatenated datasets. The model that best fits the data was determined by IQ-TREE according to the Bayesian information criterion (BIC). The LG matrix was combined to an amino acid class frequency mixture model with 20 (for single gene trees) and 40 (for concatenated datasets) frequency component profiles. Statistical support for branches was assessed by multiparametric bootstrapping (1000 replicates) in RAxML and by the ultrafast bootstrap approximation (UFboot) with 1000 replicates in IQ-TREE.

\section{Topology tests}

Phylogenetic hypotheses were tested by an approximately unbiased (AU) test [55] and expected likelihood weights (ELW) method [56] implemented in IQ-TREE 1.4.2 [54]. For all datasets we tested whether their ML phylogeny is in significant conflict with the monophyly of Metamonada. In the case of OTC and CK, we also tested whether the phylogeny is in significant conflict with the monophyly of eukaryotes. In the case of the concatenated dataset, we tested whether their ML phylogeny is in significant conflict with the monophyly of Metamonada as well as whether the relationships within the eukaryotic clade (Additional file 5) significantly conflicts with a user-defined expected species tree
(Additional file 7). For the latter two tests we used a concatenated dataset without prokaryotic sequences to eliminate the influence of relationships among prokaryotes and position of the eukaryotic root. The ADI sequence attributed to Gregarina niphandrodes was excluded from all tests including ADI data, as this clearly represents contamination or very recent LGT.

To perform AU and ELW tests, a set of 1003 topologies was created, containing the unconstrained ML topology inferred by RAxML, 1000 topologies inferred by RAxML during bootstrapping, and the best trees inferred by RAxML under the selected constraints (eukaryotic monophyly, Metamonada monophyly or eukaryotic phylogeny). Site likelihoods for topologies were calculated by IQ-TREE using the $\mathrm{LG}+\mathrm{C} 20+\mathrm{F}+\mathrm{G} 4$ model. The sets of site likelihoods were then compared using the AU test in IQ-TREE, with 10000 replicates.

\section{Cloning of ADI pathway genes}

ADI pathway genes were amplified from Paratrimastix and Monocercomonoides cDNAs by PCR. Paratrimastix pyriformis and Monocercomonoides sp. PA203 cDNAs were prepared from $100 \mathrm{~mL}$ of bacterized culture and $1000 \mathrm{~mL}$ of culture filtered according to Hampl et al. [57], respectively. Isolations of total RNA were performed using TRI Reagent RNA Isolation Reagent (Sigma, T9424). Extractions of eukaryotic mRNA from total RNA were done using a Dynabeads mRNA Purification Kit (Life Technologies, 61006). The SMARTer PCR cDNA Synthesis Kit (Clontech, 634925) was used for cDNA synthesis following by cDNA amplification with an Advantage 2 PCR Kit (Clontech, 639207) using 21 cycles (Paratrimastix) and 19 cycles (Monocercomonoides) of amplification.

The following primers were used for amplifications of full-length cDNAs of the ADI pathway genes of Paratrimastix and Monocercomonoides (restriction sites NdeI, VspI, and BamHI are in bold): Tp OTC-F (TAACATATGCCTCGCCACCTTACCAAGAT), Tp OTC-R (T AAGGATCCGTCAAGGAGGGGCTGGCCCA), Tp CKF (TAACATATGCGTATCCTCATCGCTCTCG), Tp CKR (TAAGGATCCGGCGACAATGTGGGTACCAG), PA 203 ADI-F (TAACATATGATGCAAGATATTCACGTT CC), PA203 ADI-R (TAAGGATCCCTGATTTCCCAGAGATGCTA), PA203 OTC-F (ATCATTAATATGTCCGC TCCCGTTAGACA), PA203 OTC-R (TAAGGATCCCTCAATGGTCATTTTCTTGT), PA203 CK-F (CACTTCACATTACATATGGTGAGAATTTTAATTGCTC), PA203 CK-R (CGTATGGGTAGGATCCTGGAACAATGTGAG TTCCTT). For transfection of Trichomonas vaginalis, the genes were cloned into TagVag2 plasmid vector [58] using restriction digestion and ligation, or directly using the Infusion HD Cloning Kit (Clontech, 639648) in the case of Monocercomonoides CK. Lab-grown chemically 
competent Escherichia coli XL1 cells were used for transformations with ligation mixtures, whereas Stellar competent cells (Clontech, 636763) were used for transformation with the in-fusion reactions. Bacterial clones were checked by colony PCR for the presence of the plasmids followed by sequencing of isolated plasmids.

\section{Selectable transfection of Trichomonas vaginalis}

Despite extensive efforts, we did not achieve either stable or transient transfection of Paratrimastix pyriformis and Monocercomonoides sp. PA203 with plasmid vectors specifically prepared for those two organisms (data not shown). Therefore, the Trichomonas vaginalis heterologous expression system was used to infer the subcellular localizations of the Paratrimastix and Monocercomonoides enzymes. Versions of ADI pathway genes with a Cterminal 2xHA-tag were electroporated into Trichomonas cells according to the protocol described by Sutak et al. [59]. Briefly, $250 \mathrm{~mL}$ of Trichomonas vaginalis T1 culture (strain kindly provided by Michaela Marcinčiková, Dept. of Parasitology, Charles University) was used for two electroporations performed for each of the genes. Cells were electroporated with $30 \mu \mathrm{g}$ of TagVag2 plasmid isolated using the Wizard Plus Midipreps DNA Purification System (Promega, A7640). The exponential protocol (350 V, $975 \mu \mathrm{F}, \infty \Omega, 4 \mathrm{~mm}$ cuvette) of the GenePulser Xcell Electroporation System (Biorad, 165-2660) was used for each transfection. Trichomonads were selected with $200 \mu \mathrm{g} / \mathrm{ml}$ of G418 (ZellBio, G-418-5) for at least five passages. Expression of the proteins was analyzed by Western blotting of cell homogenates (data not shown) and immunofluorescence microscopy with antibody.

\section{Immunofluorescence microscopy}

ADI pathway proteins of Paratrimastix and Monocercomonoides were identified in Trichomonas cells using an antiHA rat monoclonal antibody (Roche, 11867423001). An antibody raised against malic enzyme, a hydrogenosomal marker in Trichomonas vaginalis [60], was used for doublelabeling (antibody kindly provided by prof. Jan Tachezy, Dept. of Parasitology, Charles University). Alexa Fluor-488 goat anti-rat (green) and Alexa Fluor-594 goat anti-rabbit (red) (Life Technologies, A-11006 and A-11037) were used as secondary antibodies. Immunostaining was done according to Sagolla et al. [61] on superfrost microscopic slides coated with poly-L-lysine (Sigma, P8920). Preparations were counterstained with DAPI in Vectashield mounting medium (Vector Laboratories, $\mathrm{H}-1200$ ) and observed using a IX81 fluorescent microscope (Olympus) equipped with an IX2-UCB camera. Images were processed using $\mathrm{Cell}^{\mathrm{R}}$ software (Olympus) and Image 1.42q.

\section{Additional files}

\begin{abstract}
Additional file 1: Phylogenetic tree of OTC sequences. The tree based on a 242 positions long protein alignment of 444 sequences was constructed in RAxML using the LG4X $+\lceil$ model of substitution. Eukaryotic taxa are highlighted in different colors according to the major group they belong to. The color code is the same as in Fig. 1. The values at nodes represent RAxML bootstrap support/IQ-TREE bootstrap support. Only values above $50 \%$ are shown. Black circles indicate support of $100 \% / 100 \%$. Species with multiple sequences included: Alexandrium tamarense 1 - CAMPEP 0186340278; Alexandrium tamarense 2 - CAMPEP 0186191854; Alexandrium tamarense 3 CAMPEP 0186247540; Durinskia baltica 1 - CAMPEP 0200033980; Durinskia baltica 2 - CAMPEP 0200081736; Euglena gracilis 1 - c20598 g1 i1; Euglena gracilis 2 - c34673 g1 i6; Eutreptiella gymnastica-like 1 - CAMPEP 0200420840; Eutreptiella gymnastica-like 2 - CAMPEP 0200409666; Karenia brevis 1 CAMPEP 0188881430; Karenia brevis 2 - CAMPEP 0188950444; Karlodinium micrum 1 - CAMPEP 0200795676; Karlodinium micrum 2 - CAMPEP 0200767534. The tree is rooted with sequences of bacterial aspartate carbamoyltransferase (ATC; EC 2.1.3.2). (PDF 506 kb)
\end{abstract}

Additional file 2: Phylogenetic tree of CK sequences. The tree based on a 251 positions long protein alignment of 256 sequences was constructed in RAxML using the LG4X+ $\Gamma$ model of substitution. Eukaryotic taxa are highlighted in different colors according to the major group they belong to. The color code is the same as in Fig. 1. The values at nodes represent RAxML bootstrap support/lQ-TREE bootstrap support. Only values above $50 \%$ are shown. Black circles indicate support of $100 \% / 100 \%$. Vertical black bars indicate well-supported eukaryotic clades: Ch - Chlorophyta; Din Dinoflagellata; Dip - Diplomonadida; Pa - Parabasalia; Pr - Preaxostyla. Species with multiple sequences included: Giardia intestinalis 1 - GSB 16453; Giardia intestinalis 2 - GL50803 16453; Thalassiosira pseudonana 1 - Gl 223995860; Thalassiosira pseudonana 2 - Gl 224000745; Trichomonas vaginalis 1 - TVAG 420500; Trichomonas vaginalis 2 - TVAG 261970; Trichomonas vaginalis 3 - TVAG 420510. The tree is unrooted. (PDF $482 \mathrm{~kb}$ )

Additional file 3: Phylogenetic trees of gene partitions used for concatenation. The values at nodes represent maximum likelihood bootstrap percentages. Eukaryota highlighted in grey. Red taxon names indicate removed sequences. Positions of the particular genes in the alignment: ADI 0257, OTC 258-499, CK 500-750. The trees are unrooted. (PDF $270 \mathrm{~kb}$ )

Additional file 4: Phylogenetic tree of concatenated ADI, OTC, and CK sequences. The tree based on a 750 positions long protein alignment of 67 sequences was constructed in RAXML using LG $4 X+\Gamma$ model. Eukaryotic taxa are highlighted in different colors according to the major group they belong to. The color code is the same as in Fig. 1. The values at nodes represent RAxML bootstrap support/IQ-TREE bootstrap support. Only values above $50 \%$ are shown. Black circles indicate support $100 \% / 100 \%$. The tree is unrooted. (PDF $272 \mathrm{~kb}$ )

Additional file 5: Phylogenetic tree of concatenated ADI, OTC, and CK sequences with Bacteria and Archaea removed. The tree based on a 750 positions long protein alignment of 15 sequences was constructed in RAxML using LG $4 X+\Gamma$ model. The tree is unrooted. (PDF $2 \mathrm{~kb}$ )

Additional file 6: The probability of mitochondrial localization of ADI pathway enzymes in Monocercomonoides sp. PA203 and Paratrimastix pyriformis as predicted by TargetP and MitoProt II. (DOCX $12 \mathrm{~kb}$ )

Additional file 7: Topology of the expected species tree of eukaryotes. The tree is unrooted. (PDF $2 \mathrm{~kb}$ )

\section{Acknowledgements}

Authors would like to thank to Tomáš Pánek, Vít Céza, and Vojtěch Vacek, who provided RNA or CDNA for sequencing of Lacusteria cypriaca, the unidentified free-living trichomonad, Harpagon shusteri, and Monocercomonoides sp. PA203, as well as Qianqian Zhang and Aaron Heiss, who prepared the Trimastix marina RNAseq data.

\section{Funding}

Z. Z. and localization experiments were funded from Czech Science foundation project 13-22333P. A. K. and V. H. were supported by the project BIOCEV Biotechnology and Biomedicine Centre of the Academy of Sciences and Charles University (CZ.1.05/1.1.00/02.0109) from the European Regional Development 
Fund. I. Č., V. H. and sequencing was supported by Czech Science foundation projects P506-12-1010 and GAP506/11/1317. M. K. and P. J. K. were supported by a grant from the Tula Foundation to the Centre for Microbial Diversity and Evolution. C. W. S. was supported by Natural sciences and engineering research council of Canada - Alexander Graham Bell Canadian graduate scholarship. P. J. K., A. J. R., and A. G. B. S. are Senior Fellows of the Canadian Institute for Advanced Research. Funding bodies did not play any role in the design of the study, collection, analysis, interpretation of data and in writing the manuscript.

\section{Availability of data and materials}

Sequences were deposited in GenBank under accession numbers KT883858KT883885.

\section{Authors' contributions}

$\mathrm{VH}$ and $\mathrm{LN}$ designed the research. $\mathrm{ZZ}$ conducted the experiments. AK, MK, $\mathrm{MH}, \mathrm{CWS}, \mathrm{AGBS}, \mathrm{PJK}, \mathrm{AJR}$, and IC took part in obtaining the sequences. LN and AK conducted the phylogenetic analyses. AK conducted the topology tests. $L N$ and $V H$ wrote the manuscript. All authors read, edited, and approved the final manuscript.

\section{Competing interests}

The authors declare that they have no competing interests.

\section{Consent for publication}

Not applicable.

\section{Ethics approval and consent to participate}

Not applicable.

\section{Author details}

'Department of Parasitology, Charles University, Faculty of Science, Prague, Czech Republic. ${ }^{2}$ Department of Biochemistry and Molecular Biology, Dalhousie University, Halifax, Canada. ${ }^{3}$ Institute of Molecular Genetics, Academy of Sciences of the Czech Republic, Prague, Czech Republic. ${ }^{4}$ Department of Biology, Dalhousie University, Halifax, Canada. ${ }^{5}$ Department of Botany, University of British Columbia, Vancouver, Canada. ${ }^{6}$ Department of Zoology, Charles University, Faculty of Science, Prague, Czech Republic.

Received: 27 December 2015 Accepted: 28 September 2016 Published online: 06 October 2016

\section{References}

1. Cunin R, Glansdorff N, Piérard A, Stalon V. Biosynthesis and metabolism of arginine in bacteria. Microbiol Rev. 1986;50:314-52.

2. Marquis RE, Bender GR, Murray DR, Wong A. Arginine deiminase system and bacterial adaptation to acid environments. Appl Envir Microbiol. 1987;53: 198-200.

3. Casiano-Colón A, Marquis RE. Role of the arginine deiminase system in protecting oral bacteria and an enzymatic basis for acid tolerance. Appl Environ Microbiol. 1988;54:1318-24.

4. Ruepp A, Soppa J. Fermentative arginine degradation in Halobacterium salinarium (formerly Halobacterium halobium): genes, gene products, and transcripts of the arcRACB gene cluster. J Bacteriol. 1996;178:4942-7.

5. Linstead D, Cranshaw MA. The pathway of arginine catabolism in the parasitic flagellate Trichomonas vaginalis. Mol Biochem Parasitol. 1983;8:241-52.

6. Yarlett N, Lindmark DG, Goldberg B, Moharrami M, Bacchi CJ. Subcellular Localization of the Enzymes of the Arginine Dihydrolase Pathway in Trichomonas vaginalis and Tritrichomonas foetus. J Eukaryot Microbiol. 1994; 41:554-9.

7. Schofield PJ, Edwards MR, Matthews J, Wilson JR. The pathway of arginine catabolism in Giardia intestinalis. Mol Biochem Parasitol. 1992;51:29-36.

8. Biagini GA, Yarlett N, Ball GE, Billetz AC, Lindmark DG, Martinez MP, Lloyd D, Edwards MR. Bacterial-like energy metabolism in the amitochondriate protozoon Hexamita inflata. Mol Biochem Parasitol. 2003;128:11-9.

9. Andersson JO, Sjögren AM, Horner DS, Murphy CA, Dyal PL, Svärd SG, Logsdon JM, Ragan MA, Hirt RP, Roger AJ. A genomic survey of the fish parasite Spironucleus salmonicida indicates genomic plasticity among diplomonads and significant lateral gene transfer in eukaryote genome evolution. BMC Genomics. 2007:8:51.
10. Zhang Q, Táborský P, Silberman JD, Pánek T, Čepička I, Simpson AGB. Marine Isolates of Trimastix marina Form a Plesiomorphic Deep-branching Lineage within Preaxostyla, Separate from Other Known Trimastigids (Paratrimastix n. gen.). Protist. 2015;166:468-91.

11. Simpson AGB. Cytoskeletal organization, phylogenetic affinities and systematics in the contentious taxon Excavata (Eukaryota). Int J Syst Evol Microbiol. 2003; 53(Pt 6):1759-77.

12. Yarlett N, Martinez MP, Ali Moharrami M, Tachezy J. The contribution of the arginine dihydrolase pathway to energy metabolism by Trichomonas vaginalis. Mol Biochem Parasitol. 1996;78:117-25.

13. Morada M, Smid O, Hampl V, Sutak R, Lam B, Rappelli P, Dessì D, Fiori PL, Tachezy J, Yarlett N. Hydrogenosome-localization of arginine deiminase in Trichomonas vaginalis. Mol Biochem Parasitol. 2011:176:51-4.

14. Ringqvist E, Palm JED, Skarin H, Hehl AB, Weiland M, Davids BJ, Reiner DS, Griffiths WJ, Eckmann L, Gillin FD, Svärd SG. Release of metabolic enzymes by Giardia in response to interaction with intestinal epithelial cells. Mol Biochem Parasitol. 2008;159:85-91.

15. Stadelmann B, Hanevik K, Andersson MK, Bruserud O, Svärd SG. The role of arginine and arginine-metabolizing enzymes during Giardia - host cell interactions in vitro. BMC Microbiol. 2013;13:256.

16. Stadelmann B, Merino MC, Persson L, Svärd SG. Arginine consumption by the intestinal parasite Giardia intestinalis reduces proliferation of intestinal epithelial cells. PLoS One. 2012;7, e45325.

17. Banik S, Renner Viveros P, Seeber F, Klotz C, Ignatius R, Aebischer T. Giardia duodenalis arginine deiminase modulates the phenotype and cytokine secretion of human dendritic cells by depletion of arginine and formation of ammonia. Infect Immun. 2013;81:2309-17.

18. Schubert KR. Products of Biological Nitrogen Fixation in Higher Plants: Synthesis, Transport, and Metabolism. Annu Rev Plant Physiol. 1986;37:539-74.

19. Allen AE, Dupont $C L$, Oborník M, Horák A, Nunes-Nesi A, McCrow JP, Zheng $H$ Johnson DA, Hu H, Fernie AR, Bowler C. Evolution and metabolic significance of the urea cycle in photosynthetic diatoms. Nature. 2011;473:203-7.

20. Dagenais-Bellefeuille $\mathrm{S}$, Morse D. Putting the $\mathrm{N}$ in dinoflagellates. Front Microbiol. 2013:4:369.

21. Shafer J, Thompson JF. Arginine desimidase in Chlorella. Phytochemistry. 1968;7:391-9.

22. Laliberte $G$, Hellebust JA. Arginine utilization by Chlorella autotrophica and Chlorella saccharophila. Physiol Plant. 1990:79:57-64.

23. Sussenbach JS, Strijkert PJ. Arginine Metabolism in Chlamydomonas reinhardi. On the Regulation of the Arginine Biosynthesis. Eur J Biochem. 1969;8:403-7.

24. Laliberte G, Hellebust JA. The phylogenetic significance of the distribution of arginine deiminase and arginase in the Chlorophyta. Phycologia. 1991;30: 145-50.

25. Leliaert F, Smith DR, Moreau H, Herron MD, Verbruggen $H$, Delwiche CF, De Clerck O. Phylogeny and Molecular Evolution of the Green Algae. CRC Crit Rev Plant Sci. 2012;31:1-46.

26. Zúñiga M, Pérez G, González-Candelas F. Evolution of arginine deiminase (ADI) pathway genes. Mol Phylogenet Evol. 2002;25:429-44.

27. Fucikova K, Leliaert F, Cooper ED, Skaloud P, D'hondt S, De Clerck O, Gurgel F, Lewis LA, Lewis PO, Lopez-Bautista J, Delwiche CF, Verbruggen H. New phylogenetic hypotheses for the core Chlorophyta based on chloroplast sequence data. Front Ecol Evol. 2014;2:63.

28. Adl SM, Simpson AGB, Lane CE, Lukeš J, Bass D, Bowser SS, Brown MW, Burki F, Dunthorn M, Hampl V, Heiss A, Hoppenrath M, Lara E, le Gall L, Lynn DH, McManus H, Mitchell EAD, Mozley-Stanridge SE, Parfrey LW, Pawlowski J, Rueckert S, Shadwick L, Schoch CL, Smirnov A, Spiegel FW. The revised classification of eukaryotes. J Eukaryot Microbiol. 2012;59:429-514.

29. Emanuelsson O, Brunak S, von Heijne G, Nielsen H. Locating proteins in the cell using TargetP, SignalP and related tools. Nat Protoc. 2007;2:953-71.

30. Claros MG, Vincens P. Computational method to predict mitochondrially imported proteins and their targeting sequences. Eur J Biochem. 1996;241: 779-86.

31. Kessler E. Hydrogenase, photoreduction, and anaerobic growth. Bot Monogr Oxford. 1974;10:456-73.

32. Chávez LA, Balamuth W, Gong T. A light and electron microscopical study of a new, polymorphic free-living amoeba, Phreatamoeba balamuthi n. g., n. sp. J Protozool. 1986;33:397-404.

33. Cavalier-Smith T. The excavate protozoan phyla Metamonada Grasse emend (Anaeromonadea, Parabasalia, Carpediemonas, Eopharyngia) and Loukozoa emend. (Jakobea, Malawimonas): their evolutionary affinities and new higher taxa. Int J Syst Evol Microbiol. 2003;53:1741-58. 
34. Pánek T, Silberman JD, Yubuki N, Leander BS, Cepicka I. Diversity, Evolution and Molecular Systematics of the Psalteriomonadidae, the Main Lineage of Anaerobic/Microaerophilic Heteroloboseans (Excavata: Discoba). Protist. 2012;163:807-31.

35. Stairs CW, Eme L, Brown MW, Mutsaers C, Susko E, Dellaire G, Soanes DM, van der Giezen M, Roger AJ. A SUF Fe-S cluster biogenesis system in the mitochondrion-related organelles of the anaerobic protist Pygsuia. Curr Biol. 2014;24:1176-86

36. Atteia A, van Lis R, Tielens AGM, Martin WF. Anaerobic energy metabolism in unicellular photosynthetic eukaryotes. Biochim Biophys Acta. 2013;1827: 210-23.

37. Lepère C, Domaizon I, Hugoni M, Vellet A, Debroas D. Diversity and Dynamics of Active Small Microbial Eukaryotes in the Anoxic Zone of a Freshwater Meromictic Lake (Pavin, France). Front Microbiol. 2016;7:130.

38. Andersen RA. Biology and systematics of heterokont and haptophyte algae. Am J Bot. 2004;91:1508-22.

39. Karnkowska A, Vacek V, Zubáčová Z, Treitli SC, Petrželková R, Eme L, Novák L, Žárský V, Barlow LD, Herman EK, Soukal P, Hroudová M, Doležal P, Stairs CW, Roger AJ, Eliáš M, Dacks JB, Vlček C, Hampl V, Huynen MA, Duarte I, Szklarczyk R, Tovar J, León-Avila G, Sánchez LB, Sutak R, Tachezy J, van der Giezen M, Hernández M, Müller M, et al. A Eukaryote without a Mitochondrial Organelle. Curr Biol. 2016;26:1274-84.

40. Derelle R, Torruella G, Klimeš V, Brinkmann H, Kim E, Vlček Č, Lang BF, Eliáš M. Bacterial proteins pinpoint a single eukaryotic root. Proc Natl Acad Sci. 2015;201420657

41. Williams TA, Foster PG, Cox CJ, Embley TM. An archaeal origin of eukaryotes supports only two primary domains of life. Nature. 2013;504:231-6.

42. Spang A, Saw JH, Jørgensen SL, Zaremba-Niedzwiedzka K, Martijn J, Lind AE, van Eijk R, Schleper C, Guy L, Ettema TJG. Complex archaea that bridge the gap between prokaryotes and eukaryotes. Nature. 2015;521:173-9.

43. Burki F. The eukaryotic tree of life from a global phylogenomic perspective. Cold Spring Harb Perspect Biol. 2014;6:a016147.

44. He D, Fiz-Palacios O, Fu C-J, Fehling J, Tsai C-C, Baldauf SL. An alternative root for the eukaryote tree of life. Curr Biol. 2014;24:465-70.

45. Grigoriev IV, Nordberg H, Shabalov I, Aerts A, Cantor M, Goodstein D, Kuo A, Minovitsky S, Nikitin R, Ohm RA, Otillar R, Poliakov A, Ratnere I, Riley R, Smirnova T, Rokhsar D, Dubchak I. The genome portal of the Department of Energy Joint Genome Institute. Nucleic Acids Res. 2012:40(Database issue):D26-32

46. Keeling PJ, Burki F, Wilcox HM, Allam B, Allen EE, Amaral-Zettler LA, Armbrust EV, Archibald JM, Bharti AK, Bell CJ, Beszteri B, Bidle KD, Cameron CT, Campbell L, Caron DA, Cattolico RA, Collier JL, Coyne K, Davy SK, Deschamps P, Dyhrman ST, Edvardsen B, Gates RD, Gobler CJ, Greenwood SJ, Guida SM, Jacobi JL, Jakobsen KS, James ER, Jenkins B, et al. The Marine Microbial Eukaryote Transcriptome Sequencing Project (MMETSP): Illuminating the Functional Diversity of Eukaryotic Life in the Oceans through Transcriptome Sequencing. PLoS Biol. 2014;12:e1001889.

47. Altschul SF, Madden TL, Schäffer AA, Zhang J, Zhang Z, Miller W, Lipman DJ. Gapped BLAST and PSI-BLAST: A new generation of protein database search programs. Nucleic Acids Res. 1997;3389-3402.

48. Zubáčová Z, Novák L, Bublíková J, Vacek V, Fousek J, Rídl J, Tachezy J, Doležal P, Vlček Č, Hampl V. The Mitochondrion-Like Organelle of Trimastix pyriformis Contains the Complete Glycine Cleavage System. PLoS One. 2013;8, e55417.

49. Katoh K, Standley DM. MAFFT multiple sequence alignment software version 7: improvements in performance and usability. Mol Biol Evol. 2013;30:772-80.

50. Vaidya G, Lohman DJ, Meier R. SequenceMatrix: concatenation software for the fast assembly of multi-gene datasets with character set and codon information. Cladistics. 2011;27:171-80.

51. NovakData.zip [http://protistologie.cz/hampllab/NovakData.zip]. Accessed 4 Oct 2016.

52. Abascal F, Zardoya R, Posada D. ProtTest: Selection of best-fit models of protein evolution. Bioinformatics. 2005;21:2104-5.

53. Stamatakis A. RAxML version 8: a tool for phylogenetic analysis and postanalysis of large phylogenies. Bioinformatics. 2014;30:1312-3.

54. Nguyen L-T, Schmidt HA, von Haeseler A, Minh BQ. IQ-TREE: A Fast and Effective Stochastic Algorithm for Estimating Maximum-Likelihood Phylogenies. Mol Biol Evol. 2015:32:268-74.

55. Shimodaira $\mathrm{H}$. An approximately unbiased test of phylogenetic tree selection. Syst Biol. 2002;51:492-508

56. Strimmer K, Rambaut A. Inferring confidence sets of possibly misspecified gene trees. Proc Biol Sci. 2002;269:137-42.
57. Hampl V. Inference of the Phylogenetic Position of Oxymonads Based on Nine Genes: Support for Metamonada and Excavata. Mol Biol Evol. 2005;22: 2508-18.

58. Hrdy I, Hirt RP, Dolezal P, Bardonová L, Foster PG, Tachezy J, Embley TM. Trichomonas hydrogenosomes contain the NADH dehydrogenase module of mitochondrial complex I. Nature. 2004;432:618-22.

59. Sutak R, Dolezal P, Fiumera HL, Hrdy I, Dancis A, Delgadillo-Correa M, Johnson PJ, Müller M, Tachezy J. Mitochondrial-type assembly of FeS centers in the hydrogenosomes of the amitochondriate eukaryote Trichomonas vaginalis. Proc Natl Acad Sci U S A. 2004;101:10368-73.

60. Drmota T. Iron-ascorbate cleavable malic enzyme from hydrogenosomes of Trichomonas vaginalis: purification and characterization. Mol Biochem Parasitol. 1996:83:221-34

61. Sagolla MS, Dawson SC, Mancuso JJ, Cande WZ. Three-dimensional analysis of mitosis and cytokinesis in the binucleate parasite Giardia intestinalis. J Cell Sci. 2006;119:4889-900.

\section{Submit your next manuscript to BioMed Central and we will help you at every step:}

- We accept pre-submission inquiries

- Our selector tool helps you to find the most relevant journal

- We provide round the clock customer support

- Convenient online submission

- Thorough peer review

- Inclusion in PubMed and all major indexing services

- Maximum visibility for your research

Submit your manuscript at www.biomedcentral.com/submit
) Biomed Central 